\title{
Sustainable healthcare within the strategy of regional development
}

\author{
Michail Nekrasov ${ }^{1}$, Svetlana Kalina ${ }^{1}$, Svetlana Veprentsova $^{1}$, and Yulia Kekteeva ${ }^{1}$ \\ ${ }^{1}$ Departament of Psychotherapy, Pirogov Russian National Research Medical University, \\ Ostrovityanova st., 1 Moscow, Russian Federation
}

\begin{abstract}
This article has an aim of connecting the sustainable healthcare to the environmentally-friendly strategy of regional development. We describe how a growing interest in ways health organisations and systems measure and report on their sustainability is recorded in the recent years. In addition, we note the growing need for sustainability indicators in the health system, such as health insurance coverage, quality of care, and access to health services. Our results show that environmental pollution and other hazardous activities have a profound effect on human health and therefore create a burden for the healthcare system. In addition, we discuss the importance of sustainable healthcare for the health and well-being of patients and the environment. In the same time, we stress that high standards of healthcare should be preserved and maintained in spite of the reduction in health costs and environmental impacts as a part of the sustainable regional development strategies.
\end{abstract}

\section{Introduction}

Currently, many countries around the world aim to build a safer, lower-carbon health system by using effective tools and approaches. They are integrating their health systems into their efforts to achieve the World Health Organization's (WHO) Sustainable Development Goals (SDGs) [1, 2]. Performance measurements for external accountability, quality indicators have been identified by a number of organizations focused on improving health care, which are used as aggregate measurements for performance purposes or as design mechanisms to support internal quality-improvement processes, such as the World Health Organization (WHO) and the United Nations. Guidelines on the performance and sustainability of the health system were considered relevant to the main areas of interest, as they cover a wide range of aspects of health reform, including the development and implementation of measures to improve sustainability and the management of health systems $[3,4]$. Those guidelines that do not contain material content relating to sustainability and the interventions involved will be excluded. Documents that only relate to aspects related to health care - systemic reforms, such as health management, are also excluded. International documents are those that cover a wide range of aspects of the health system, such as health management and health system management, while national documents are national, and have a national scope in OECD high-income countries. To take account of this margin, it is

\footnotetext{
*Corresponding author: nekrasovma77@yandex.ru
} 
essential to have a clear understanding of what could produce further progress in the area of global health. When it comes to the role of national health systems in developing countries in the development of the health system, there are is a insufficient focus on policies and implementation gaps. As a result, more and more initiatives are being taken to strengthen national health systems as a core component of a global health system [5]. In order to respond to this challenge, numerous instruments and framework conditions have been created to increase longevity.

The experience might differ all around the world. Individuals and Unite States health organizations are using local performance measurements to support the development of a national health system in the United States and other countries. System-wide sustainability reporting and reporting on environmental sustainability are also important. This suggests that this area should be high on the list of areas where consideration should be given to how well system-wide sustainability reports can be developed to support accountability and improvement. There is the need to achieve a high level of transparency and accountability in reporting on quality performance in health systems $[6,7]$. The idea that environmental sustainability should be included as an explicit aspect of quality is not new, but this approach seems to have taken hold. Sustainability should be considered in all areas of quality and as a core part of, and should have been included in, the quadruple goal, or a key element of, a health system's sustainability strategy. Meanwhile, the link to sustainability is clear: health and health systems minimize the harm to human health they cause through pollution, but it does not yet appear to be accepted as the core of the economy. Sustainability of the health system in the region and how it has developed in recent years, both within and outside the health system. After introducing environmental ethics locally and joining the Environmental HealthAlliance, a non-profit health organization, the clinic has improved its sustainability ranking and achieved higher efficiencies. The tests and screenings of patients are carried out in-house, which makes them more efficient and cheaper for the health system. There is a high level of cooperation between medical staff and patients in the community and within the hospital [8].

The good news is that the health sector can play a leading role in solving these problems. The health industry is pressing for these concerns to be given higher priority in the current situation. With its mission - the interest in disease prevention - the health sector can help to move the entire economy toward sustainable, safe products and practices. Health Care without Harm is a global initiative to transform the medical waste incineration industry in the United States and worldwide, endangering patient safety and care and responsible for more than $60 \%$ of global waste. Without Harm began in 2010 after medical waste incineration was identified as the leading source of greenhouse gas emissions from the United States and Canada and by 2014 was identified by the United States Department of Health as a major cause of global climate change, while medical waste incinerators were identified as one of the three main sources of global warming $[9,10]$.

\section{Recent challenges for sustainable healthcare}

One can understand the importance of critical health literacy as a key component of the health system. If we fail to go beyond the basic communication problems discussed above, we can compare it to a health-care system that focuses only on emergency medicine, rather than addressing the problem at its root [11]. After all, health literacy is the ability of individuals to take care of their own health and to effect change through community action. In the clinical field, authorities also begun to consider how efforts to strengthen patient literacy and health literacy could be stepped up.

The evidence suggests that health organizations are evolving to strengthen coordination of primary and secondary care. There is a need for a population - based on the perspective of 
the role of health - that learns literacy in the health system, and for better coordination between primary and secondary care [12].

Health care managers and executives also face the challenge of providing primary health referral services to meet highly complex, changing needs that often lead to resource wastage. These trends are challenged by front and middle management work, which is limited in capacity, and by lack of time. In the face of the pace of change, organisations must be flexible and offer high-quality care at low cost. However, the potential for widespread demand in the health system could limit providers' ability to shift resources to emerging trouble spots. During the early coronavirus pandemic in spring 2020, providers relied on high-quality, lowcost primary and emergency care to manage demand, and shifted elective care to free up resources for COVID-19 patients [13]. However, a recent increase in hospital admissions shows that this pressure will increase in the coming months and years, with an increase in demand for care, supported by the need for intensive care units and more frequent hospital stays. Indeed, health analysis has the potential to reduce treatment costs, predict outbreaks and epidemics, prevent preventable diseases, and improve quality of life in general. Moreover, the average human lifespan is increasing and the world population is facing new challenges to today's treatment and delivery methods.

Given the unique challenges that are developing in each country, health systems and public health leaders need to adapt quickly to prevent potential COVID-19-related crises and save lives [14]. Just like entrepreneurs, doctors are able to collect vast amounts of data and seek out the best strategies to use these numbers. One can look at the examples of big data in health care that already exist and that medical institutions can benefit from. It is also important to note that this can include not only hospitals, but also health care providers, medical schools and other medical institutions [15].

The medical field can be difficult and stressful, but with the right tools, healthcare providers can help employees feel better about their work and work more confidently. Health care personnel are supposed to improve the quality of life of their employees. The persons entrusted with the management and support of doctors and nurses and medical staff. The system of healthcare cannot be expected to perform at the top level in rare crises, and many of these challenges become more visible as the tide turns against the health-care system [16].

There always challenges of the time in the health sector, particularly with regard to the quality of life of health workers. Now that all eyes are on these challenges, it is time to discuss them with our colleagues in the health and health sectors, as well as in the public and private sectors, and to share them with certainty. Paying the price for the shortcomings of the first days and the long-term health system as a whole, for shortcomings that go beyond the first days. Patients and families often report that caregivers do not coordinate their work or even know what the other is doing. Patients spend a lot of time moving from centre to centre, consulting with nurses who are too busy and therefore not working enough. Overlapping and contradictory treatments that are costly, confusing and, worse, detrimental to patients [17].

In some recent studies, physicians surveyed said that uncoordinated treatment resulted in one or more negative outcomes, and more than half said that the lack of coordination was usually due to patients receiving conflicting health information from providers. Broadly speaking, these challenges could include possible disclosure requirements in relation to positive test results. For example, widespread testing could show that asymptotic health care workers care for patients, which provides a way to react negatively to COVID-19, allowing them to stay at work [18]. All of the above constitute some recent challenges for the healthcare system that need to be overcame and settled. 


\section{Financing regional healthcare systems}

Healthcare funding has evolved from personal payments to financing from the time-ofservice delivery to personal payment and from financing to health insurance [19]. Government funding is also needed for services that insurance plans avoid or are inefficiently accessible, including community services such as mental health and drug abuse services. There is a problem in the United States, where government funding comes from the federal government, state and local governments, private insurance companies, and the private sector [20]. From government funding, we move on to supplementing private health insurance and other forms of health care with government funding. To prevent oversupply of private insurance plans for the provision of health services, regulation of additional government funding is needed. When multiple agencies are involved in a system that grants direct government grants, there is often a conflict of interest between those who may need the most help and those who need it. This is a major financial burden on public health services, which are generally underfunded compared to clinical services. In such circumstances, public health services very often orient themselves towards excluding people from health services because they lack health insurance. This is particularly true in areas such as mental health and drug abuse, as well as the treatment of children and adolescents [21].

In 2018, China has established a new central government health insurance authority that will manage the three major health insurance companies, which are also harmonized. Public financing is one of many countries that have a mixed public financing system [22]. China, Mongolia, the Philippines, and Vietnam have recently undergone, or already have undergone, changes in health-care reforms. At national level, the benefits are defined by their fund group and the number of benefits covered as well as the costs of the benefits. Generally, there is limited coverage for preventive care, but there are a number of other benefits, such as dental, visual and mental health care. The health insurance also partially covers the costs of primary care for children under 18 years of age and offers access to a wide range of outpatient services and a range of prevention services. Children and adolescents only have a limited part of the insurance cover. To fund health care, each region and municipality levies proportionate income taxes on its population. More than two-thirds of the region's total revenue came from local taxes, and subsidies and state grants are financed by national income taxes and indirect taxes. The state grant will be used to redistribute the funds between municipalities and regions as needed.

National health expenditure is derived from both government and non-government sources and used to finance a wide range of programs and services. In the United States. health care funding includes raising money for health care through taxes, subsidies, grants and other sources such as health insurance, Medicare and Medicaid expansion, and private insurance. The federal government's share of the national health budget ( $\$ 1.2$ trillion in 2016) accounts for less than 1 percent of all health spending. The whole system is characterised by competition for resources, which is reflected in the distribution of money and the provision of services. Priorities for health care funding in each region of the United States, as well as at the state and local levels are indicated. The lack of a mechanism to protect against financial risk and disease leads to a vicious circle of disease and poverty. There is progress towards equity in financial protection, but there is little evidence that it works [23]. The relatively large impact of the poor has had a relatively larger impact on them, as even small payments can make up a significant part of their budget. Only six countries in the Americas have World Health Organization figures, and their average annual health spending ranges from $4 \%$ to $64 \%$. This protects the population from the risk of impoverished and catastrophic health spending. Egypt's health system is pluralistic and fragmented in terms of funding, sources of funding, and financing mechanisms. The typology of the country's wealth-financing system consists of a mix of public and private sources of finance, earmarked health taxes, and public- 
private partnerships (PPP). Its dominance is alarmingly high in some countries like, for example, Egypt, with five sources playing an important role in financing health spending: public health, private health insurance, government subsidies, tax revenues, public subsidies, and private-sector subsidies.

\section{Healthcare budgets and spending}

There is a reverse share of spending on health care for people with certain conditions, while the country has largely ignored other factors that affect people's health. Recent estimates put 10 to 20 percent of health outcomes down to medical care, and the other 80 to 90 percent to health-related behaviours [24]. We know that many developing countries spend proportionately more on social services than the United States, but because medical spending in some countries is a higher percentage of GDP than in other developed countries, it is harder to compare spending on SDH. This comparison only gives an overview of the proportional spending in each country, as social services do not correspond and many other factors do not. At the state level, North Carolina is trying to transform its entire Medicaid system by prioritizing social determinants, but at a cost of $\$ 1.2$ billion a year. Beginning in 2019, North Carolina plans to screen all Medicaid recipients who enrol in managed care for diseases such as diabetes, heart disease, cancer and diabetes. Local health care providers are also launching medical pilot programs to combat social determinants and, ideally, reduce expensive perpatient care. Some groups of people who also report poor health are medically underserved and may have health problems that cannot be solved without expensive medical services. Regardless, health status alone may not always be the best indicator of expected health care spending in the year in question. Patients are assigned to a range of social determinants, including race, ethnicity, gender, education, age, income and education. These organisations share referrals to provide more accurate information about their patients' health status and outcomes [25].

The importance of United State health spending has continued to grow, now accounting for more than $17 \%$ of the US economy. Little is known about the size and growth of these expenditures, however, and how expenditures on these conditions vary according to age and time. The average annual cost of health care in the United States in 2015 was $\$ 1,832$, up from $\$ 2,071$ in 2010. A number of officials collected and combined data on health spending on diseases such as diabetes, high blood pressure, heart disease, cancer and obesity. Official estimates of National Health Expenditure (NHEA) include total health spending in two administrative cost categories associated with health care services, such as health insurance, prescription drugs, and medical devices. In addition to estimating the expenditure on personal health care, the study also produced preliminary estimates reflecting the state-funded public health expenditure of the states and municipalities as well as the federal government. Personal health expenditure (89\%), which accounted for 5\% of total health expenditure in 2013, was the focus of this study and was defined in NHEA as total expenditure on the treatment of people with specific diseases [26].

Starting in 2017, 19 U.S. states require Medicaid to screen recipients for social needs and transfer enrolment to social services. Six U.S. states also require sergeants to coordinate care when an inmate has left custody, and other states plan to implement such requirements in 2018. A recent survey of more than 1,000 providers of care programs found that nearly all of the recipients surveyed (91\%) said that activities that take into account social factors such as employment, housing, education and employment status were considered to be key components of social and health activities [27]. 


\section{Conclusions}

Overall, the principles of sustainable health care can contribute to the delivery of sustainable clinical practice by reducing wasteful health activities and requirements and reducing the environmental impact of remaining health activities. One cannot underestimate the importance of reducing health costs, waste and environmental impacts while maintaining a high standard of care.

Many organizations developed guidelines to evaluate existing health systems and to consider sustainability in the design and redesign of care models. As we make progress towards sustainable financing in this area, it is important to take a practical and workable approach that is in line with budgetary and financial considerations. By working with local communities and minimizing the environmental footprint of medicine, sustainable healthcare's goal is to be part of an economy that best serves patients and society and protects the environment. We must come together to ensure global health and to take integrated measures to protect and support people and the planet.

At the municipal level, reducing the environmental footprint in hospitals conserves natural resources and reduces environmental risks. Green building planning is done with contractors who agree with sustainability principles and green-going-green construction process for all of hospitals and facilities. A model is needed that reduces environmental pollution by reducing health and wasteful activities, as well as carbon intensity. The first steps towards sustainable practices can be done by emphasizing the importance of using fewer resources for reuse and recycling, rethinking and exploring responsibility and taking responsibility. This sustainability assessment applies to the purchase of medical devices, which generate about half a billion dollars in annual spending. Given the importance of sustainable healthcare for the health and well-being of patients and the environment, it is crucial that the profession makes use of the opportunities offered by the new sustainable healthcare guidelines to address this issue in the earliest stages of medical education. Medical students and junior doctors need to be involved in analysing current practice, improving their understanding of the environmental impact of their practice, and presenting possible changes to their colleagues in clinics and management. Medical students and junior doctors have the opportunity to participate in the analysis and improvement process and improve their current practices and present possible changes to their clinical, senior and colleagues. In order to truly incorporate sustainability into our health culture, we must work to integrate it into the education and training of health professionals. Embedding sustainability principles in medical education will normalize a broader thinking about sustainability and its role in health care.

\section{References}

1. T. Hone, J. Macinko, C. Millett, The Lancet, 392(10156), 1461-1472 (2018)

2. M.E. Cerf, Global Challenges, 3(9), 1900021 (2019)

3. J. Bejtkovský, Marketing and Management of Innovations, 1, 294-302 (2020)

4. S. Kalyugina, W. Strielkowski, L. Ushvitsky, E. Astachova, Journal of Security \& Sustainability Issues, 5(2), 297-304 (2015)

5. T. Oraro-Lawrence, K. Wyss, BMC health services research, 20(1), 1-11 (2020)

6. J. Braithwaite, P. Hibbert, B. Blakely, J. Plumb, N. Hannaford, J.C. Long, D. Marks, SAGE open medicine, 5, 2050312116686516 (2017)

7. A.S. Fauci, R.R. Redfield, G. Sigounas, M.D. Weahkee, B.P. Giroir, Jama, 321(9), 844845 (2019)

8. D. Cepiku, F. Giordano, T. Bovaird, E. Loeffler, Public Money \& Management, 41(1), 77-80 (2021) 
9. C. Thiel, P. Duncan, N. Woods, Journal of health services research \& policy, 22(3), 162167 (2017)

10. S. Ilyas, R.R. Srivastava, H. Kim, Science of the Total Environment, 749, 141652 (2020)

11. P. Dunn, S. Conard, International journal of cardiology, 273, 249-251 (2018)

12. S. Vamos, O. Okan, T. Sentell, I. Rootman, International journal of environmental research and public health, 17(4), 1436 (2020)

13. D. Golinelli, E. Boetto, G. Carullo, A.G. Nuzzolese, M.P. Landini, M.P. Fantini, Journal of medical Internet research, 22(11), e22280 (2020)

14. M. Casale, Global Public Health, 15(11), 1740-1752 (2020)

15. S. Dash, S.K. Shakyawar, M. Sharma, S. Kaushik, Journal of Big Data, 6(1), 1-25 (2019)

16. H. Blake, F. Bermingham, G. Johnson, A. Tabner, International journal of environmental research and public health, 17(9), 2997 (2020)

17. M.C. Kok, J.E. Broerse, S. Theobald, H. Ormel, M. Dieleman, M. Taegtmeyer, Human resources for health, 15(1), 1-7 (2017)

18. K.M. Stepien, B. Kieć-Wilk, C. Lampe, T. Tangeraas, G. Cefalo, N. Belmatoug, M. Scarpa, Frontiers in Medicine, 8, 197 (2021)

19. K.J. Caswell, T.A. Waidmann, Medical Care Research and Review, 76(5), 538-571 (2019)

20. M. Hartman, A.B. Martin, J. Benson, A. Catlin, Health Affairs, 39(1), 8-17 (2020)

21. N.T. Douthit, S. Biswas, Frontiers in public health, 6, 114 (2018)

22. X. Li, H.M. Krumholz, W. Yip, K.K. Cheng, J. De Maeseneer, Q. Meng, S. Hu, The Lancet, 395(10239), 1802-1812 (2020)

23. T. Rice, L.Y. Unruh, E. Van Ginneken, P. Rosenau, A.J. Barnes, Health Policy, 122(7), 698-702 (2018)

24. R.G. Watt, B. Daly, P. Allison, L.M. Macpherson, R. Venturelli, S. Listl, H. Benzian, The Lancet, 394(10194), 261-272 (2019)

25. A. Dunn, P. Shieh, L. Fernando, C. Roehrig, Spending by Condition for the LongTerm Care Population Using Medicaid Claims, https://www.bea.gov/system/files/papers/BEA-WP2020-10.pdf(2020)

26. P. Osterman, Who Will Care for Us? Long-term Care and the Long-term Workforce (2017)

27. E. Byhoff, L.A. Taylor, American journal of preventive medicine, 57(6), 74-81 (2019) 